\title{
Corrigendum
}

\section{Corrigendum to "Wind and Payload Disturbance Rejection Control Based on Adaptive Neural Estimators: Application on Quadrotors"}

\author{
Jesús Enrique Sierra $\mathbb{D}^{1,2}$ and Matilde Santos $\mathbb{D}^{1}$ \\ ${ }^{1}$ Computer Science Faculty, Complutense University of Madrid, 28040 Madrid, Spain \\ ${ }^{2}$ Department of Civil Engineering, Area of Languages and Computer Systems, University of Burgos, 09006 Burgos, Spain \\ Correspondence should be addressed to Jesús Enrique Sierra; jesierra@ubu.es and Matilde Santos; msantos@ucm.es \\ Received 27 January 2020; Accepted 28 January 2020; Published 24 August 2020 \\ Copyright ( ) 2020 Jesús Enrique Sierra and Matilde Santos. This is an open access article distributed under the Creative Commons \\ Attribution License, which permits unrestricted use, distribution, and reproduction in any medium, provided the original work is \\ properly cited.
}

In the article titled "Wind and Payload Disturbance Rejection Control Based on Adaptive Neural Estimators: Application on Quadrotors" [1], an affiliation was omitted in error. This affiliation has been added to the affiliation list above as number 2 , and the author affiliations have been corrected.

\section{References}

[1] J. E. Sierra and M. Santos, "Wind and payload disturbance rejection control based on adaptive neural estimators: application on quadrotors," Complexity, vol. 2019, Article ID 6460156, 20 pages, 2019. 